\title{
Distúrbios do sono e fatores associados a transtornos psíquicos menores em indivíduos em tratamento oncológico
}

\author{
Sleep disorders and factors associated with minor psychic disorders in individuals under \\ oncological treatment \\ Trastornos del sueño y factores asociados con trastornos psíquicos menores en personas en \\ tratamiento oncológico
}

Bruna Matos Santos Dantas

ORCID: https://orcid.org/0000-0002-2829-8422

Universidade Estadual de Feira de Santana, Brasil

E-mail: brunamatos.dantas@gmail.com

Maria Emília Cirqueira Silva

ORCID: https://orcid.org/0000-0002-8556-482X

Faculdade Anísio Teixeira, Brasil

E-mail: emiliacirqueira@gmail.com

Lorena Moura de Assis Sampaio

ORCID: https://orcid.org/0000-0003-0627-4494

Universidade Estadual de Feira de Santana, Brasil

E-mail: lorenamoura@fat.edu.br

Lucas Souza Almeida de Araujo

ORCID: https://orcid.org/0000-0001-5723-6725

Faculdade Anísio Teixeira, Brasil

E-mail: lucas.araujx@hotmail.com

Guthierre Almeida Portugal

ORCID: https://orcid.org/0000-0003-1779-4997

Universidade Estadual de Feira de Santana, Brasil

E-mail: guthierresamu@gmail.com

Magno Conceição das Mercês

ORCID: https://orcid.org/0000-0003-3493-8606

Universidade do Estado da Bahia, Brasil

E-mail:magnomerces@hotmail.com

Aron José Brito de Miranda

ORCID: https://orcid.org/0000-0003-4712-7415 Instituto Federal de Educação, Ciência e Tecnologia da Bahia, Brasil

E-mail: aronjose@gmail.com

Julita Maria Freitas Coelho

ORCID: https://orcid.org/0000-0002-9520-5177

Instituto Federal de Educação, Ciência e Tecnologia da Bahia, Brasil

E-mail: julitamaria@gmail.com

\begin{abstract}
Resumo
O câncer constitui-se como umas das morbidades que mais assolam a população em todo o mundo e é a causa de morte de milhões de pessoas. Nesse sentido, o presente estudo teve por objetivo detectar associações entre distúrbios do sono e outros fatores aos Transtornos Psíquicos Menores (TPM) em indivíduos com câncer atendidos em uma clínica especializada no interior da Bahia, Brasil. Realizou-se um estudo transversal com 205 indivíduos diagnosticados com algum tipo de câncer, que estavam sendo atendidos na Unidade de Alta Complexidade em Oncologia (UNACON), em que foi traçado o perfil sociodemográfico, de condição de saúde e estilo de vida dos participantes a partir de um formulário por meio de entrevista. $\mathrm{O}$ desfecho foi determinado conforme a presença do Transtorno Psíquico Menor (TPM). Foram obtidas frequências simples e relativas e a comparabilidade entre os grupos foi feita com o teste quiquadrado de Pearson ou Exato de Fisher, com nível de significância de 5\%. Houve o predomínio do sexo feminino, prevalência de TPM de 63,44\% entre as mulheres $(\mathrm{p}=0,04)$, sedentarismo, baixa escolaridade, renda e presença de doença crônica instalada. O estudo demonstrou associação positiva e estatisticamente significante entre a insônia e o transtorno psíquico menor, além de outros fatores encontrados que se associam a ocorrência desses transtornos. Tal
\end{abstract}


inquérito foi realizado entre indivíduos em tratamento antineoplásico, que pode ser considerado mais um fator para agravos físicos e psicológicos na vida dessas pessoas.

Palavras-chave: Câncer; Transtorno psíquico menor; Distúrbios do sono; Estilo de vida.

\begin{abstract}
Cancer is one of the morbidities that most affect the population worldwide and is the cause of death for millions of people. In this sense, the present study aimed to detect associations between sleep disorders and other factors with Minor Psychic Disorders (PMD) in individuals with cancer treated at a specialized clinic in the interior of Bahia, Brazil. A cross-sectional study was carried out with 205 individuals diagnosed with some type of cancer, who were being treated at the High Complexity Unit in Oncology (UNACON), in which the sociodemographic profile, health condition and lifestyle of the participants was drawn. from a form through an interview. The outcome was determined according to the presence of Minor Psychic Disorder (MPD). Simple and relative frequencies were obtained and comparability between groups was performed using Pearson's chi-square test or Fisher's exact test, with a significance level of 5\%. There was a predominance of females, prevalence of PMS of $63.44 \%$ among women $(\mathrm{p}=0.04)$, sedentary lifestyle, low education, income and presence of installed chronic disease. The study showed a positive and statistically significant association between insomnia and minor mental disorder, in addition to other factors found that are associated with the occurrence of these disorders. This survey was carried out among individuals undergoing antineoplastic treatment, which can be considered another factor for physical and psychological harm in these people's lives.
\end{abstract}

Keywords: Cancer; Minor psychic disorder; Sleep disorders; Lifestyle.

\title{
Resumen
}

El cáncer es una de las morbilidades que más afecta a la población a nivel mundial y es la causa de muerte de millones de personas. En este sentido, el presente estudio tuvo como objetivo detectar asociaciones entre los trastornos del sueño y otros factores con los Trastornos Psíquicos Menores (PMD) en individuos con cáncer atendidos en una clínica especializada en el interior de Bahía, Brasil. Se realizó un estudio transversal con 205 individuos diagnosticados de algún tipo de cáncer, que se encontraban en tratamiento en la Unidad de Alta Complejidad en Oncología (UNACON), en el que se extrajo el perfil sociodemográfico, estado de salud y estilo de vida de los participantes. un formulario a través de una entrevista. El resultado se determinó de acuerdo con la presencia de trastorno psíquico menor (MPD). Se obtuvieron frecuencias simples y relativas y la comparabilidad entre grupos se realizó mediante la prueba de chicuadrado de Pearson o la prueba exacta de Fisher, con un nivel de significancia del 5\%. Predominó el sexo femenino, prevalencia de SPM del 63,44\% entre las mujeres $(\mathrm{p}=0,04)$, sedentarismo, baja escolaridad, ingresos y presencia de enfermedad crónica instalada. El estudio mostró una asociación positiva y estadísticamente significativa entre el insomnio y el trastorno mental menor, además de otros factores encontrados que están asociados con la aparición de estos trastornos. Esta encuesta se realizó entre individuos sometidos a tratamiento antineoplásico, lo que puede considerarse un factor más de daño físico y psicológico en la vida de estas personas.

Palabras clave: Cáncer; Trastorno psíquico menor; Trastornos del sueño; Estilo de vida.

\section{Introdução}

O câncer constitui-se como umas das morbidades que mais assolam a população em todo o mundo e é a causa de morte de milhões de pessoas, com incidência e mortalidade em aumento global. Na última estimativa mundial, 18 milhões de novos casos e 9,6 milhões de óbitos foram contabilizados. Só no Brasil, o Instituto Nacional do Câncer José de Alencar da Silva (INCA) estima-se para o biênio 2020-2022 a ocorrência de 650 mil casos novos da doença, sendo próstata e mama os mais prevalentes, respectivamente, em homens e mulheres, sem considerar os cânceres de pele não melanoma. Se for considerado o cálculo global corrigido para subregistro (Mathers et al., 2003), a estimativa alcança os 685 mil novos casos (INCA, 2015).

Além disso, uma transição daqueles com etiologia associada à melhoria das condições socioeconômicas com a incorporação de hábitos e atitudes associados à urbanização (sedentarismo, alimentação inadequada, entre outros) comparados com os casos relacionados a infecções (Bray et al., 2018). Não só o alto índice é preocupante, mas as consequências trazidas pelas neoplasias na vida dos indivíduos diagnosticados com a doença, tais como pensamentos negativos e incertezas (Mattias et al., 2018) e alterações do sono e fadiga advindos da quimioterapia (Ferreira et al., 2017; Whisenant et al., 2017).

Ou seja, no indivíduo acometido por câncer é comum ter-se a presença de sintomas que evidenciem sofrimento mental, pois sentimentos de temor, angústia e estresse estão diretamente ligados à situação. No entanto, deve-se diagnosticar o momento em que esses sintomas se agravam, levando aos transtornos mentais, que precisam ser tratados. Depressão e ansiedade, por 
exemplo, influenciam no curso da doença, já que pessoas com esses transtornos tem dificuldade para tomar decisões, seguir o tratamento e, portanto, perdem a qualidade de vida (Crespo et al., 2007).

Os Transtornos Psíquicos Menores (TPM), que também recebem a nomenclatura de Transtornos Mentais Comuns (TMC) (Goldberg et al., 1992), não se classificam como doença mental, pois não obedecem a todos os critérios diagnósticos contidos na Classificação Internacional de Doenças (CID 10), mas sim, como um quadro de sintomas a exemplo de insônia, irritabilidade, tristeza, preocupação somática, diminuição da concentração e fadiga. No entanto, os sintomas apresentados se comparam ou até superam os crônicos de doença mental já estabelecida (Kac et al., 2006).

Dentre os sintomas evidenciados, um dos problemas mais frequentes observados em pacientes oncológicos está relacionado ao sono. O sono é uma função biológica fundamental na consolidação da memória, na visão binocular, na termorregulação, na conservação e restauração da energia e restauração do metabolismo energético cerebral (Reimão, 1996; Ferrara et al., 2001). Devido a essas importantes funções, as perturbações do sono podem acarretar alterações significativas no funcionamento físico, ocupacional, cognitivo e social do indivíduo, além de comprometer substancialmente a qualidade de vida (Crespo et al., 2007)

Os distúrbios do sono, vivenciados pelos pacientes oncológicos, podem ocorrer em diferentes momentos: no diagnóstico, durante e após o tratamento e na fase terminal. A incidência desse distúrbio para esses pacientes é significativa, pois gira em torno de 30 a 50\%, quando comparado aos 15\% na população em geral. Além disso, de 23 a $44 \%$ dos pacientes oncológicos apresentam esse sintoma após dois a cinco anos de tratamento (Goldberg et al., 1992).

Tendo em vista a relação entre sofrimento mental, câncer e diversos fatores envolvidos na perda da qualidade de vida dos indivíduos acometidos pela doença, como distúrbios no sono, esses problemas não devem ser acompanhados separadamente. Entender o ser humano e como ele funciona não deve ser feito de forma fragmentada por áreas específicas do conhecimento (Kac et al., 2006), apontando a interdisciplinaridade como a base do tratamento das neoplasias, perpassando por cada etapa da evolução patológica.

Nesse sentido, o presente estudo teve por objetivo detectar associações entre distúrbios do sono e outros fatores aos Transtornos Psíquicos Menores (TPM) em indivíduos com câncer atendidos em uma clínica especializada no interior da Bahia, Brasil.

\section{Metodologia}

Desenvolveu-se pesquisa de campo, do tipo corte transversal. Fizeram parte do estudo 205 indivíduos diagnosticados com algum tipo de câncer, que estavam sendo atendidos na Unidade de Alta Complexidade em Oncologia (UNACON) em Feira de Santana- Bahia, no período da coleta de dados (maio a agosto de 2015). A participação destes foi voluntária mediante assinatura do Termo de Consentimento Livre e Esclarecido (TCLE).

A coleta de dados ocorreu no período de maio a agosto de 2015, após aprovação do Comitê de Ética e Pesquisa da Faculdade Anísio Teixeira (FAT), parecer de n 1.065.062/ CAE: 40390615.0.0000.5631. Foi aplicado um questionário, preenchido pelo pesquisador sob comando verbal do participante. O conteúdo do questionário foi dividido em blocos de perguntas separadas por quatro temas: identificação; aspectos sócio-demográficos; biologia humana; hábitos de vida e saúde geral. A condição da insônia/ distúrbio do sono foi obtida através do quesito quantidade de horas dormida. Se menor que 8 horas, os participantes foram incluídos no grupo exposto.

Para avaliação de TPM, utilizou-se o Self- Report Questionare- SQR, que contém 20 itens, para respostas objetivas (SIM ou NÃO), sobre problemas que podem ter ocorrido nos últimos 30 dias, como dores de cabeça, tristeza, dificuldades para tomar decisão, entre outros. Foram considerados com TPM todos aqueles que responderam positivamente ao mínimo de sete questões inseridas no questionário supracitado. 
Todos os dados foram processados e categorizados utilizando-se o programa SPSS 17.0 for Windowns- Statistical Package for Social Science 2000 e STATA versão 11.0. Inicialmente, foi procedida a caracterização da população estudada com a obtenção de frequências simples e frequências relativas das variáveis categóricas de interesse. Em seguida, foi realizada a análise bivariada, estimando a Razão de Prevalência (RP) e seus respectivos Intervalos de Confiança (IC) e valores de p pelos testes Qui quadrado de Pearson ou Exato de Fisher, com nível de significância de 5\%. Por fim, foi empregada regressão logística não consicional para obter um modelo final de associação entre insônia e o transtorno psíquico menor, ajustado para as variáveis candidatas a confundidoras.

\section{Resultados e Discussão}

A amostra estudada incluiu 205 indivíduos com câncer, atendidos numa Unidade de Alta Complexidade em Oncologia (UNACON) no interior do estado baiano. O emprego de questionário possibilitou a obtenção das características sociodemográficas dos indivíduos estudados apresentadas na tabela 1. Os dados obtidos corroboram com achados (Reimão, 1996), em que observaram maior concentração de pessoas do sexo feminino e residentes na zona urbana.

Houve o predomínio do sexo feminino ( $\mathrm{N}=114)$ e prevalência de TPM $63,44 \%$ neste grupo, evidenciando uma associação estatisticamente significante $(\mathrm{p}=0,04)$. A literatura aponta que os TPM estão mais presentes no sexo feminino, pessoas com mais idade, indivíduos pretos e pardos, menor nível de instrução e baixa renda, tabagistas e pessoas acometidas por doenças crônicas (Ferrara et al., 2001; Muller et al., 2007). Estes resultados são semelhantes aos apresentados (Tabela 1), enfatizando a importância da observação dos grupos descritos, com o objetivo de detectar precocemente a instalação dos transtornos psíquicos menores, que embora tidos como muito comuns, têm suas repercussões comparadas ou até superam as de doenças mentais orgânicas (Juver et al., 2008).

Tabela 1 - Características sociodemográficas e de estilo de vida e transtornos psíquicos menores em pacientes em tratamento oncológico. Feira de Santana, BA, Brasil, 2015.

\begin{tabular}{lccc}
\hline & \multicolumn{3}{c}{ Transtornos Psíquicos Menores } \\
Características & $\begin{array}{c}\text { Sim (n=93) } \\
\mathbf{n}(\%)\end{array}$ & $\begin{array}{c}\text { Não (n=112) } \\
\mathbf{n}(\%)\end{array}$ & $\mathbf{p}^{*}$ \\
Idade (n=203) & & & \\
$\leq 60$ anos & $56(60,87)$ & $35(31,53)$ & $<0,00$ \\
$>60$ anos & $36(39,13)$ & $76(68,47)$ & \\
Sexo $(\mathbf{n = 2 0 5 )}$ & & & \\
Feminino & $59(63,44)$ & $55(49,11)$ & 0,04 \\
Masculino & $34(36,56)$ & $57(50,89)$ & \\
Raça/Cor da pele (n=205) & & & \\
Branco & $16(17,20)$ & $18(16,07)$ & 0,82 \\
Não branco & $77(82,80)$ & $94(83,93)$ & \\
Situação conjugal (n=205) & & & \\
Com parceiro & $56(60,22)$ & $70(62,50)$ & 0,73 \\
Sem parceiro & $37(39,78)$ & $42(37,50)$ & \\
Nível de escolaridade (anos de estudo) $(\mathbf{n = 2 0 5})$ & & & \\
Maior que 4 anos de estudo & $32(34,41)$ & $40(35,71)$ & 0,84 \\
Até 4 anos de estudo & $61(65,59)$ & $72(64,29)$ & \\
\hline
\end{tabular}

\footnotetext{
Renda (salário mínimo) $(\mathrm{n}=\mathbf{2 0 5})$
} 


$\begin{array}{llll}\geq 1 \text { salário mínimo } & 18(19,35) & 29(25,89) & 0,26 \\ <1 \text { salário mínimo } & 75(80,65) & 83(74,11) & \\ \mathbf{N}^{\mathbf{0}} \text { de filhos (n=205) } & & & \\ \text { Até } 3 \text { filhos } & 50(53,76) & 54(48,21) & 0,42\end{array}$

Mais que 3 filhos

Local de residência $(n=205)$

Zona urbana

Zona rural

Cidade de residência $(\mathbf{n}=\mathbf{2 0 5})$

Feira de Santana

Outras

Ocupação $(\mathbf{n = 2 0 5})$

Com ocupação

Sem Ocupação

Rede de esgoto $(\mathbf{n}=\mathbf{2 0 5})$

Sim

Não

Atividade física $(\mathrm{n}=\mathbf{2 0 5})$

Sim

Não

Hábito de fumar (n=205)

Sim

Não

Consumo de bebida alcóolica $(\mathrm{n}=\mathbf{2 0 5})$

Sim

Não

\section{Horas de Sono $(\mathbf{n}=205)$}

$\geq 8$ horas por dia

$<8$ horas por dia
$43(46,24)$

$58(51,79)$

$\begin{array}{lll}65(69,89) & 74(66,07) & 0,56 \\ 28(30,11) & 38(33,93) & \end{array}$

$$
\begin{array}{ll}
59(63,44) & 70(62,50) \\
34(36,56) & 42(37,50)
\end{array}
$$

0,89

$\begin{array}{lll}11(11,83) & 14(12,50) & 0,88 \\ 82(88,17) & 98(87,50) & \end{array}$

$\begin{array}{lll}55(59,14) & 71(63,39) & 0,53 \\ 38(40,86) & 41(36,61) & \end{array}$

$\begin{array}{lll}14(15,05) & 40(35,71) & 0,00 \\ 79(84,95) & 72(64,29) & \end{array}$

* Nível de significância estatística: $p \leq 0,05$.

Fonte: Pesquisa de Campo (2015).

A maior parte da amostra foi composta por idosos com idade igual ou superior a 60 anos $(55,17 \%)$, sendo que a idade geral variou dos 18- 91 anos. Quando analisadas as idades segundo a presença ou não de TPM (tabela 1), identificou-se uma ocorrência maior desses transtornos nos indivíduos com idade $\leq 60$ anos $(60,87 \%)$, com diferenças estatisticamente significantes $(\mathrm{p}<0,00)$. Esses resultados divergem dos encontrados nos estudos que descreveram prevalências maiores desses transtornos em indivíduos entre 55 - 64 anos e a partir dos 65 anos (Juver et al., 2008). No entanto, já haviam descrito uma prevalência menor de TPM em pessoas com mais de 60 anos de idade (Souza et al., 2012). Deve-se destacar que os estudos citados neste parágrafo foram a partir de amostras que não estudavam diretamente pessoas em tratamento antineoplásico, dificultando as comparações entre os estudos.

Foi realizado um levantamento da prevalência de transtornos mentais comuns (TMC) em idosos na mesma cidade deste estudo e utilizando o mesmo instrumento, o SQR-20, que revelou uma porcentagem de 32,1\% de TMC nos idosos entrevistados, 
sendo que entre os praticantes de atividades físicas como parte de lazer esse número foi menor, evidenciando a associação de sedentarismo e menos momentos de lazer com presença de TMC (Araújo et al., 2005).

Esses dados se relacionam com os apresentados neste inquérito quanto às características da amostra, tendo em comum faixa etária, predomínio do sexo feminino, prevalência de sedentarismo, baixa escolaridade, baixa renda e presença de doença crônica instalada, indicando que tais características podem favorecer a presença de TPM não só nos grupos específicos de idosos e indivíduos acometidos pelo câncer, mas também na população em geral, necessitando mais estudos nessa perspectiva. Salientase que nesta investigação detectou-se diferença estatisticamente significante entre prática de atividade física e sofrimento mental de acordo com os escores do SQR-20 ( $\mathrm{p}=0,00)$.

Ao analisar a renda dos indivíduos entrevistados, pode-se constatar uma discrepância nas frequências, caracterizando a amostra em economicamente baixa, já que 77\% apresentou renda inferior a um salário mínimo. Quando feita a comparação entre os grupos com e sem TPM (tabela 1), os dados mostraram que apenas 19\% dos indivíduos com TPM possuíam renda $\geq 1$ salário mínimo. Embora essas diferenças não tenham sido significantes estatisticamente $(\mathrm{p}=0,26)$, esses resultados estão de acordo com os já encontrados na literatura, que destacam as baixas condições financeiras nos indivíduos com TPM (Kirchhof et al., 2009).

Em relação aos hábitos de fumar e consumo de álcool, a maioria dos entrevistados relataram fazer ou já terem feito uso de bebidas alcoólicas e tabaco, 66,82\% e 55,60\%, respectivamente, nos dois grupos de comparação (com e sem TPM) (Tabela 1), não havendo diferenças significantes. Em contraste, um inquérito realizado na mesma cidade desse estudo com o objetivo de estimar a prevalência de transtornos mentais comuns nessa população, detectou que tabagistas tem 2,14 vezes mais chances de desenvolverem TPM do que aqueles que não fumam ( $\mathrm{RP}=2,14$; IC- 1,64 - 2,79), no entanto, não houve associação para o consumo de álcool, assim como em outras pesquisas com o mesmo objetivo (Maragno et al., 2006).

Tabela 2 - Características relacionadas à saúde geral da amostra, de acordo com presença ou ausência de TPM. Feira de Santana, BA, Brasil, 2015.

\begin{tabular}{|c|c|c|c|}
\hline \multirow[b]{2}{*}{ Características } & \multicolumn{2}{|c|}{ Transtornos Psíquicos Menores } & \multirow[b]{2}{*}{$\mathbf{p}^{*}$} \\
\hline & $\begin{array}{c}\operatorname{Sim}(\mathbf{n}=93) \\
\mathrm{n}(\%)\end{array}$ & $\begin{array}{c}\text { Não }(n=112) \\
n(\%)\end{array}$ & \\
\hline \multicolumn{4}{|l|}{ Diabetes $(n=205)$} \\
\hline Não & $79(84,95)$ & $94(83,93)$ & 0,84 \\
\hline Sim & $14(15,05)$ & $18(16,07)$ & \\
\hline \multicolumn{4}{|c|}{ Doença Cardiovascular ( $\mathrm{N}=205)$} \\
\hline Não & $78(83,87)$ & $102(91,07)$ & 0,12 \\
\hline Sim & $15(16,13)$ & $10(8,93)$ & \\
\hline \multicolumn{4}{|c|}{ Doença Renal (n=205) } \\
\hline Não & $87(93,55)$ & $100(89,29)$ & 0,28 \\
\hline $\operatorname{Sim}$ & $06(6,45)$ & $12(10,71)$ & \\
\hline \multicolumn{4}{|c|}{ Doença Pulmonar (n=205) } \\
\hline Não & () & () & 0,05 \\
\hline Sim & () & () & \\
\hline \multicolumn{4}{|c|}{ Doença Hepática (n=205) } \\
\hline Não & $83(89,25)$ & $105(93,75)$ & 0,24 \\
\hline Sim & $10(10,75)$ & $07(6,25)$ & \\
\hline \multicolumn{4}{|c|}{ Hipertensão (n=205) } \\
\hline Não & $47(50,54)$ & $59(52,68)$ & 0,76 \\
\hline
\end{tabular}




\begin{tabular}{|c|c|c|c|}
\hline Sim & $46(49,46)$ & $53(47,32)$ & \\
\hline \multicolumn{4}{|c|}{ Infecção (n=205) } \\
\hline Não & $90(96,77)$ & $108(96,43)$ & 0,89 \\
\hline Sim & $03(3,23)$ & $04(3,57)$ & \\
\hline \multicolumn{4}{|c|}{ Acidente Vascular Cerebral $(n=205)$} \\
\hline Não & $87(93,55)$ & $110(98,21)$ & 0,09 \\
\hline Sim & $06(6,45)$ & $02(1,79)$ & \\
\hline \multicolumn{4}{|c|}{ Alergia $(n=205)$} \\
\hline Não & $71(76,34)$ & $91(81,25)$ & 0,39 \\
\hline Sim & $22(23,66)$ & $21(18,75)$ & \\
\hline
\end{tabular}

* Nível de significância estatística: $\mathrm{p} \leq 0,05$;

Fonte: Pesquisa de Campo (2015).

A Tabela 2 apresenta as características relacionadas à saúde geral da amostra, tendo a hipertensão arterial sistêmica com a maior prevalência. Esse dado está de acordo com os encontrados na literatura, vez que estudos demonstram que hipertensão e diabetes são as doenças mais frequentemente encontradas na população adulta e idosa (Andrade et al., 1999). Deve-se atentar que presença de doenças crônicas associadas, a exemplo de hipertensão e câncer, como mostrou esse inquérito, são causas de altos graus de incapacidade em indivíduos com mais idade, tornando imprescindível que as equipes que prestam cuidados a esse público estejam cada vez mais atentas e atualizadas técnico e cientificamente, objetivando promover acolhimento e cuidados mais efetivos a esses clientes.

A condição da insônia foi obtida através do quesito, quantidade de horas dormidas. Se menor que 8 horas, os participantes foram incluídos no grupo exposto, que apresentou um percentual de 61,29\% de TPM, revelando uma associação estatisticamente significante ( $\mathrm{p}<0,00)$ (tabela 1). Para tal associação, após utilizar o método de regressão logística, estimou-se que a probabilidade dos que não dormem pelo menos oito horas por dia apresentar TPM foi de 2,74 vezes maior do que aqueles que relataram dormir no mínimo oito horas, com significância estatística $(\mathrm{OR}=2,74 ; \mathrm{IC} 95 \%=1,55-4,83$; $\mathrm{p}=0,043)($ Tabela $3)$.

Os dados reafirmam o estudo que demonstrou que indivíduos que apresentavam dificuldade para dormir $(\mathrm{RP}=4,17$; 1,18 - 14,77) e os que acordavam no meio da noite e tinham dificuldade para voltar a dormir $(\mathrm{RP}=5,69 ; 1,43$ - 22,75) exibiam prevalências muito maiores de TMC comparando com os que não apresentavam distúrbios do sono (Andrade et al., 1999).

Sobre esta associação, a combinação entre psicopatologia e sofrimento psíquico em distúrbios do sono é bidirecional (Rocha et al., 2010), na medida em que, tanto os transtornos mentais afetam a ocorrência de distúrbios do sono, quanto estes estão relacionados à ocorrência de transtornos mentais. No presente trabalho, partiu-se do pressuposto de que o câncer altera muitos aspectos da vida do indivíduo e pode trazer modificações expressivas ao seu cotidiano, inclusive em relação à qualidade do sono (Alves et al., 2007). Este por sua vez, influenciará no desenvolvimento e/ou exacerbação do TMC. 
Tabela 3 - Associação bruta entre duração do sono e transtorno psíquico menor em indivíduos em tratamento oncológico. Feira de Santana, BA, Brasil, 2015.

\begin{tabular}{lccccc}
\hline Duração do sono & $\begin{array}{c}\text { Com TPM } \\
\mathbf{n}(\%)\end{array}$ & $\begin{array}{c}\text { Sem TPM } \\
\mathbf{n}(\%)\end{array}$ & OR $^{*}$ & IC 95\% & ${\text { Valor de } \boldsymbol{p}^{* *}}^{\text {(\%) }}$ \\
\hline < 8 horas & $57(61,29)$ & $41(36,61)$ & 2,74 & $1,55-4,83$ & 0,043 \\
$\mathbf{8}$ 8 horas & $36(38,71)$ & $71(63,39)$ & - & - & - \\
\hline
\end{tabular}

*Odds Ratio Bruta

** Nível de significância estatística: $\mathrm{p} \leq 0,05$

Fonte: Autores.

\section{Conclusão}

O estudo demonstrou associação positiva e estatisticamente significante entre a insônia e o transtorno psíquico menor, além de outros fatores encontrados que se associam a ocorrência desses transtornos. Tal inquérito foi realizado entre indivíduos em tratamento antineoplásico, que pode ser considerado mais um fator para agravos físicos e psicológicos na vida dessas pessoas.

Salienta-se que por se tratar de um estudo de corte transversal, causa e efeito não podem ser estabelecidas, logo, para as associações encontradas destaca-se a necessidade de mais estudos nessa temática, que possibilitem acréscimos no conhecimento científico, e assim, aumento na qualidade dos cuidados oferecidos a esses pacientes.

Como os serviços de oncologia são relativamente novos no cenário da cidade de Feira de Santana, Bahia, observa-se a relevância do estudo, na medida em que a identificação de questões como essas poderão contribuir no desenvolvimento de estratégias para este grupo, a fim de buscar melhorias na qualidade do sono, e, por conseguinte reduzir a prevalência deste tipo de transtorno que tanto influencia na qualidade de vida.

Em suma, com base nos fatos mencionados anteriormente, evidencia-se a importância do presente estudo gerar a realização de mais pesquisas, com o escopo de ampliar ainda mais o conhecimento acerca dos distúrbios do sono e fatores associados a transtornos psíquicos menores.

\section{Referências}

Alves, L. C., Leimann, B. C. Q., Vasconcelos, M. E. L., Carvalho, M. S., Vasconcelos, A. G. G., Fonseca, T. C. O da F., Lebrão, M. L \& Laurentti, R. (2007). A influência das doenças crônicas na capacidade funcional dos idosos do Município de São Paulo, Brasil The effect of chronic diseases on functional status of the elderly living in the city of São Paulo, Brazil. Cad. Saúde Pública, 23, 1924-1930. http://www.scielo.br/pdf/\%0D/csp/v23n8/19.pdf.

Amorim, J. R., Silva, I. A. \& Shimizu, I. S. (2017). Avaliação da qualidade do sono em pacientes com câncer de mama em quimioterapia. Rev Bras Mastologia 27(1):3-7. http://www.rbmastologia.com.br/wp-content/uploads/2017/01/MAS-v27n1_3-7.pdf 10.5327/Z201700010002RBM.

Andrade, L. H. S. G., Lolio, C. A, Gentil, V. \& Laurenti, R. (1999). Epidemiologia dos transtornos mentais em uma área definida de captação da cidade de São Paulo, Brasil. Rev. psiquiatr. clín. 26, 257-61. http://bases.bireme.br/cgi-bin/wxislind.exe/iah/online/?IsisScript=iah/iah.xis\&src=google\&base=ADO LEC\&lang=p\&nextAction $=$ lnk\&exprSearch=256441\&indexSearch=ID.

Araújo, T. M., Pinho, P de S. \& Almeida, M. M. G de. (2005). Prevalência de transtornos mentais comuns em mulheres e sua relação com as características sociodemográficas e o trabalho doméstico. Rev. bras. saúde matern. Infant. 5, 337-348. http://www.scielo.br/scielo.php?script=sci _arttext\&pid=S1519_ 38292005000300010

Bray, F., Ferlay, J., Soerjomataram, I., Siegel, R. L., Torre, L. A. \& Jemal, A. (2020) Global cancer statistics 2018: GLOBOCAN estimates of incidence and mortality worldwide for 36 cancers in 185 countries. CA Cancer J Clin. 2018 68(6):394-424. 10.3322/caac.21492. Erratum in: CA Cancer J Clin. 70(4):313. Global cancer statistics 2018: GLOBOCAN estimates of incidence and mortality worldwide for 36 cancers in 185 countries

Chaoul, A., Milbury, K., Spelman, A., Basen-Engquist, K., Hall, M. H. \& Wei, Q., et al. (2017). Randomized trial of Tibetan yoga in patients with breast cancer undergoing chemotherapy. Cancer 124(1):36-45. https://onlinelibrary.wiley.com/doi/epdf/10.1002/cncr.30938.

Crespo, A de S. \& Lourenço, M. T da C. (2007). No impacto psicológico da doença. In: Mohallem, A. G. da C.; Rodrigues, A. B. (Orgs.). Enfermagem Oncológica. Barueri: Manole, 141-148.

Estrela, C. (2018). Metodologia Científica: Ciência, Ensino, Pesquisa. Editora Artes Médicas. 
Ferrara, M. \& Gennaro, L de. (2001). How much sleep do we need?. Sleep medicine reviews, 5, 155-179. http://www.sciencedirect.com/science/article/pii/S1087079200901382.

Ferreira, R. G. R. \& Franco, L. F. R. (2017). Efeitos colaterais decorrentes do tratamento quimioterápico no câncer de mama: revisão bibliográfica. Rev Univ Vale Rio Verde 15(2):633-8. Efeitos Colaterais Decorrentes Do Tratamento Quimioterápico No Câncer De Mama: revisão bibliográfica (researchgate.net).

Goldberg, D. \& Huxley, P. (1992) Common mental disorders: a bio-social model. Tavistock/Routledge; 194p. A bio-social model for common mental disorders - PubMed (nih.gov).

Horta, B. L. \& Victora, C. G. (2008). Prevalência e determinantes precoces dos transtornos mentais comuns na coorte de nascimentos de 1982, Pelotas, RS. Rev Saúde Publica, 42, 26-33. http://www.scielo.br/pdf/rsp/v42s2/7002.pdf.

INCA, Instituto Nacional de Câncer José Alencar Gomes da Silva. (2015). Coordenação de Prevenção e Vigilância. Estimativa 2016: Incidência de Câncer no Brasil. INCA, www.inca.gov.br/wcm/dncc/2015/dados-apresentados.pdf>.

Juver, J. P da S. \& Verçosa, N. (2008). Depressão em pacientes com dor no câncer avançado. Revista Brasileira de Anestesiologia. 58, http://www.scielo.br/pdf/rba/v58n3/en_12.pdf .

Kac, G., Silveira, E. A., Oliveira, L. C de M. \& Jesus, J de. (2006). Fatores relacionados a prevalência de morbidades psiquiátricas menores em mulheres selecionadas em um Centro de Saúde no Rio de Janeiro, Brasil. Cad. de Saúde Pública. 22, 999-07. http://www.scielo.br/pdf/csp/v22n5/12.pdf.

Kirchhof, A. L. C., Magnago, T. S. B de S., Camponogara, S., Griep, R. H., Tavares, J. P., Prestes, F. C. \& Paes, L. G. (2009). Condições de Trabalho e características sócio-demográficas relacionadas à presença de Distúrbios Psíquicos Menores em trabalhadores de enfermagem. Texto Contexto Enfermagem. 18, 215-23. http://www.scielo.br/pdf/tce/v18n2/03.pdf.

Koche, J. C. (2011). Fundamentos de metodologia científica. Petrópolis: Vozes. Disponível em: http://www.brunovivas.com/wpcontent/uploads/sites/10/2018/07/K\%C3\%B6che-Jos\%C3\%A9-Carlos0D0AFundamentos-de-metodologia-cient\%C3\%ADfica-_-teoria-da0D0Aci\%C3

\%AAnci a-e-inicia\% C3\%A7\%C3\%A3o-\%C3\%A0-pesquisa.pdfhttps://repositorio.ufsm.br/bitstream/handle/1/15824/Li c_Computacao_Metodologia-Pesqu isa-Cientific a.pdf? sequence $=1$

Lamino, D. A., Pimenta, C. A. M., Braga, P. E. \& Mota, D. D. C. F. (2015). Fadiga clinicamente relevante em mulheres com câncer de mama: prevalência e fatores associados. Investig Enferm [Internet]. Jan [acesso em 2018 jun 24];17(1):65-76. http://www.redalyc.org/html/1452/145233516005.

Ludke, M. \& Andre, M. E. D. A. (2013). Pesquisas em educação: uma abordagem qualitativa. E.P.U.

Mansano-Schlosser, T.C. \& Ceolim, M.F. (2012). Fatores associados à qualidade do sono de idosos submetidos à quimioterapia. Revista Latino-Americana de Enfermagem, 20, 1100-1108. http://www.periodicos.usp.br/rlae/article/viewFile/52907/56860.

Maragno, L., Goldbaum, M., Gianini, R. J., Novaes, H. M. D. \& Chester, L. G. C. (2006). Prevalência de transtornos mentais comuns em populações atendidas pelo Programa Saúde da Família (QUALIS) no município de São Paulo, Brasil. Cad. de Saúde Pública. 22, 1639-48. http://www.scielo.br/pdf/csp/v22n8/12.pdf.

Martins, L. C. X. \& Kuhn, L. (2013). Prevalência de transtornos mentais comuns em jovens brasileiros recém-incorporados ao Serviço Militar Obrigatório e fatores associados. Ciênc. saúde coletiva, 18, 1809-1816. https://www.researchgate.net/profile/Lilian_Martins/publication/237097050_Prevale nce_of_common_mental_disorders_in_recently-drafted_young_Brazilians_to_mand atory_military_service_and_associated_factors/links/0deec520cce363e678000000.pdf.

Mathers, C. D., Bernard, C., Iburg, K. M., Inoue, M., Fat, D. M., Shibuya, K., Stein, C., Tomijima, N. \& Xu, H. (2003). Global burden of disease in 2002: data sources, methods and results. Geneva: WHO. (Global programme on evidence for health policy discussion paper, v. 54). Microsoft Word - paper54.doc (psu.edu).

Mattias, S. R., Pinto, K. R. T da F., Lima, N de M., Santos, I. D. de., Bernardy, C. C. F. \& Sodré, T. M. (2018). Câncer de mama: sentimentos e percepções das mulheres diante do diagnóstico. Revista Online de Pesquisa, 10(2):385-390. Câncer de mama: sentimentos e percepções das mulheres diante do diagnóstico | Rev. Pesqui. 10(2): 385-390

Muller, M. R. \& Guimarães, S. S. (2007). Impacto dos transtornos do sono sobre o funcionamento diário e a qualidade de vida. Estud. psicol.(Campinas), p. 519-528. http://www.scielo.br/scielo.php?script=sci_arttext\&pid=S0103-166X2007000400011.

Pereira A. S. et al. (2018). Metodologia da pesquisa científica. UFSM.

Reimão, R. (1996. Sono: estudo abrangente. (2a ed.), Atheneu.

Rocha, S. V., Almeida, M. M. G, Araújo, T. M. de. \& Virtuoso Júnior, J. S. (2011). Atividade física no lazer e transtornos mentais comuns entre idosos residentes em um município do Nordeste do Brasil. J Bras Psiquiatr, 60, 80-85. http://www.scielo.br/pdf/jbpsiq/v60n2/02.pdf.

Rocha, S. V., Almeida, M. M. G, Araújo, T. M de. \& Virtuoso Júnior, J. S. (2010). Prevalência de transtornos mentais comuns entre residentes em áreas urbanas de Feira de Santana, Bahia. Rev Bras Epidemiol, 13, 630-40. https://www.researchgate.net/profile/Jair_Virtuoso_ Junior/publication/49704756_Prevalence_of_common_mental_disorders_among_the_residents_of_urban_areas_in_Feira_de_Santana_Bahia/links/556bdbc20 8aec22683037e41.pdf.

Sateia, M. J. (2009). Update on sleep and psychiatric disorders. CHEST Journal, 135, 1370-1379. http://journal.publications.che stnet.org/article.aspx?articleid=108 9816\#Insomnia.

Souza, R. S., Simão. D. A da S. \& Lima, E. D. R de P. (2012). Perfil sociodemográfico e clínico de pacientes atendidos em um serviço ambulatorial de quimioterapia paliativa em Belo Horizonte. Revista Mineira de Enfermagem, 16, 38-47. http://reme.org.br/artigo/detalhes/498.

Vachani, C. (2007). Insomnia in the patient with cancer. Oncolink-Abramson Cancer Center of the University of Pennsylvania http://www.oncolink.com/coping/article.cfm?c=5\&s=69\&ss=116\&id=709. 
Research, Society and Development, v. 10, n. 8, e46210817703, 2021

(CC BY 4.0) | ISSN 2525-3409 | DOI: http://dx.doi.org/10.33448/rsd-v10i8.17703

Whisenant, M., Wong, B., Mitchell, S. A., Beck, S. L. \& Mooney, K. (2017). Distinct Trajectories of Fatigue and Sleep Disturbance in Women Receiving Chemotherapy for Breast Cancer. Oncol Nurs Forum. 44(6):739-750. Distinct Trajectories of Fatigue and Sleep Disturbance in Women Receiving Chemotherapy for Breast Cancer

Yin, R. K. (2015). O estudo de caso. Bookman. 